\title{
Simulación de áreas de alta consecuencia para gasoductos
}

\author{
Simulation of high consequence areas for gas pipelines
}

\author{
Simulação de áreas de alta consequência para gasodutos
}

\author{
Orlando Díaz-Parra ${ }^{1}$ \\ Enrique Vera-López ${ }^{2}$
}

Recibido: septiembre de 2017

Aceptado: noviembre de 2017

Para citar este artículo: Díaz-Parra, O., y Vega-López, E. (2018). Simulación de áreas de alta consecuencia para gasoductos. Revista Científica, 31(1), 32-44. Doi: https://doi.org/10.14483/23448350.12513

\section{Resumen}

El gasoducto es utilizado para el transporte de gas natural a gran distancia. Los riesgos derivados del manejo de un material combustible transportado a alta presión por conducciones que pasan cerca del lugar donde habitan personas hace necesario que se adopten medidas de prevención, mitigación y control para disminuir el efecto en caso de ignición de una fuga de gas. En este trabajo se muestra el desarrollo de un nuevo modelo matemático para determinar áreas de alta consecuencia y su aplicación mediante software de amplia disponibilidad y fácil manejo, como lo son Google Earth y Excel, para determinar y visualizar el área en la cual el nivel de radiación puede afectar la integridad de las personas y las edificaciones. El modelo tiene en cuenta la caída de presión al interior del gasoducto a partir de la estación de compresión, el índice de escape de gas y las formas posibles de ignición del gas. Este desarrollo se constituye en una alternativa ante el uso de software especializado y personal altamente capacitado. La simulación se aplica a un trazado del gasoducto Miraflores-Tunja utilizando una macro desarrollada en Excel para determinar el área de impacto y compararla con las coordenadas de las áreas vulnerables. Las zonas en las que se intersectan se constituyen en áreas de alta consecuencia y se identifican junto con los tramos del gasoducto que las afectan para entregar al operador una herramienta de análisis de riesgo para la determinación y visualización del gasoducto y su entorno.

Palabras clave: análisis de riesgo, áreas de alta consecuencia, gasoducto, gas natural, integridad, simulación.

\begin{abstract}
The gas pipeline is used for the transport of natural gas at a great distance. Risks derived from the handling of a combustible material transported under high pressure, by pipelines that pass close to where people live, makes it necessary to adopt prevention, mitigation and control measures to reduce the effect in case of ignition of a gas leak. This work shows the development of a new mathematical model to determine areas of high consequence and their application, using widely available and easy to use software, such as Google Earth and Excel, to determine and visualize the area up to which the level of
\end{abstract}


radiation can affect the integrity of people and buildings. The model take into account the pressure drop into the gas pipeline from the compression station, the gas leakage rate and possible forms of gas ignition. This development is an alternative to the use of specialized software and highly trained personnel. The simulation is applied to a traced of the Miraflores-Tunja gas pipeline, using a macro developed in Excel to determine the impact area and compare it with the coordinates of the vulnerable areas. The zones where these areas intersect are constituted in high consequence areas and are identified along with the sections of the pipeline that affect them, to provide the operator with an risk analysis tool for the determination and visualization of the gas pipeline and its environment.

Keywords: gas pipelines, high consequence areas, integrity, natural gas, risk analysis, simulation.

\section{Resumo}

O gasoduto é usado para o transporte de gás natural a uma grande distância. Os riscos derivados do manuseio de um material combustível transportado sob alta pressão, por condutas que passam perto de onde as pessoas vivem, torna necessário adotar medidas de prevenção, mitigação e controle para reduzir o efeito em caso de ignição de vazamento de gás. Este trabalho mostra o desenvolvimento de um novo modelo matemático para determinar áreas de alta conseqüência e sua aplicação usando um software amplamente disponível e fácil de usar, como Google Earth e Excel, para determinar e visualizar a área até a qual o nível de pode afetar a integridade de pessoas e edifícios. O modelo leva em consideração a queda de pressão a partir da estação de compressão, a taxa de escape de gás e as possíveis formas de ignição do gás. Este desenvolvimento é uma alternativa ao uso de software especializado e pessoal altamente treinado. Simulação é aplicada a uma rota do gasoduto Miraflores-Tunja, usando uma macro desenvolvida no Excel para determinar a área de impacto e compará-la com as coordenadas das áreas vulneráveis. Nas áreas onde essas áreas se cruzam constituem as áreas de alta conseqüência e são identificados juntamente com os trechos do gasoduto que os afetam, para fornecer ao operador uma ferramenta de análise de risco para a determinação e visualização do gasoduto e seu arredores.

Palavras-chaves: análise de risco, áreas de alta conseqüência, gasoduto, gás natural, integridade, simulação.

\section{Introducción}

La delimitación de áreas de alta consecuencia (AAC) en un gasoducto se realiza teniendo en cuenta los factores de riesgo derivados de la naturaleza del fluido, el volumen de fluido transportado, la presión interna aplicada al fluido, la dimensión y material del tubo conductor, el entorno físico-geográfico por donde transita y del entorno social y poblacional circundante de dichas instalaciones (Daza-Leguizamón, Vera-López y Riaño-Cano, 2016).

Son de reconocida aplicabilidad en la delimitación o determinación de las AAC normas nacionales (NTC-5747, 2009) e internacionales (API-581 2002; Code of Federal Regulations, 2017; GRI-00/0189, 2000). Del mismo modo, se han realizado numerosas investigaciones al respecto (Ma et al., 2013; Jo y Ahn, 2002; Hui y Guoning, 2012; Tong et al., 2013; Li y Huang, 2012; Park, Lee y Jo 2004) las cuales concluyen en modelos simplificados para el cálculo del radio de impacto o área de consecuencia en la que son factores importantes la presión de operación, el diámetro de la conducción, el índice de escape de gas y el umbral de radiación que se considera admisible. La realización de un análisis de riesgo lleva al operador de un gasoducto a tomar las medidas necesarias para prevenir, mitigar o controlar los factores de riesgo en las zonas que sean definidas como AAC.

En este trabajo se muestra la aplicación de un nuevo modelo matemático para determinar las AAC únicamente en función de la distancia a la estación de compresión, la cual considera las posibles pérdidas de vidas humanas, las pérdidas económicas y en la propiedad derivados de la fuga de gas natural y su posible ignición, así como también la forma de expansión de los gases, las 
características de la mezcla aire-gas y la forma en que se desarrolla el proceso de ignición.

La aplicación se realiza mediante la simulación computacional del modelo desarrollado con herramientas informáticas de amplia utilización y fácil manejo, como lo son Google Earth y Excel, para determinar e identificar las AAC sobre un trazado escogido como caso de estudio. El resultado es la visualización del área de impacto potencial y la localización de las AAC y los tramos del gasoducto que las afectan, para que el usuario final, o el operador del gasoducto, identifique las áreas en las que deberá adoptar medidas de mitigación y control.

El modelo desarrollado se aplicó a un caso de estudio correspondiente a un tramo de un gasoducto en Colombia (gasoducto Miraflores-Tunja), para el que se identificó un trazado y áreas de ocupación humana. Mediante el diseño de macros en Excel se ejecutan órdenes en lazo cerrado y se procesan datos en una hoja de cálculo para obtener archivos que, llevados a mapas geográficos informáticos en Google Earth, permiten visualizar el área de impacto y las AAC.

\section{Metodología}

A continuación, se exponen algunos conceptos básicos sobre el gas natural y las AAC para continuar con los modelos de delimitación existentes. Después se muestra el desarrollo del nuevo modelo matemático de delimitación de AAC y se describe el diseño del proceso de simulación del mismo desarrollado por medio de las herramientas de Google Earth y Excel.

\section{El gas natural y la AAC}

El gas natural es una mezcla de hidrocarburos livianos en estado gaseoso que en su mayor parte está compuesta por metano y etano, y en menor proporción por propano, butanos, pentanos e hidrocarburos más pesados. El gas natural es inflamable y explosivo (Huang y Li, 2012). La densidad relativa del gas natural es 0,58 , mucho menor que la del aire. Cuando se fuga gas natural al aire y su fracción en volumen en la mezcla está entre 5,3\% a $15 \%$ se puede producir la combustión (Hui y Guoning 2012).

El término ACC se refiere a una zona que se extiende a cada lado de un gasoducto que pasa por áreas de ocupación humana permanente, como poblaciones o conjuntos residenciales, o transitorias, como iglesias, hospitales o colegios, en un entorno urbano o suburbano. Un AAC se delimita mediante la construcción de un área de impacto y la verificación de la existencia de áreas vulnerables dentro de esta zona, como se muestra en la figura 1.

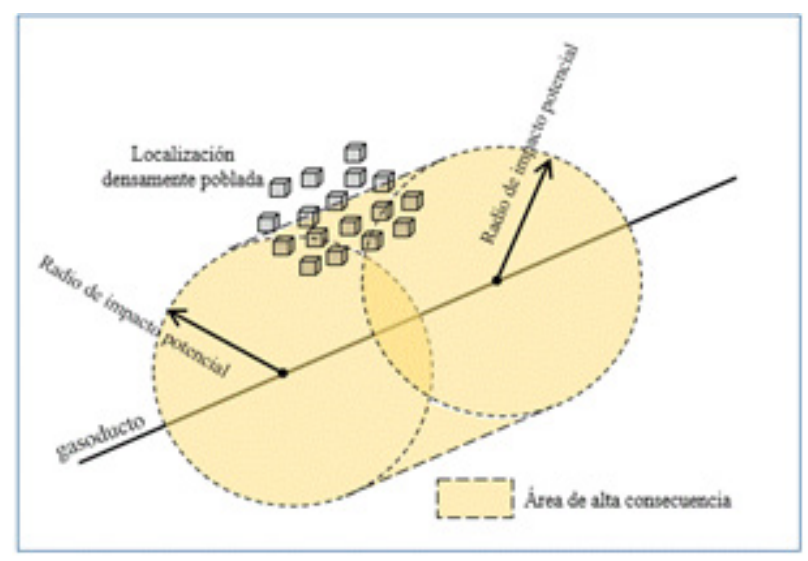

Figura 1. Extensión del área de alta consecuencia cuando el círculo de impacto potencial contiene una localización vulnerable.

Fuente: apéndice E, CFR-49-142.

La información presentada en investigaciones previas sobre carga térmica sugiere que no se espera que una estructura de madera se queme por debajo de $15 \mathrm{~kW} / \mathrm{m} 2$ (Committee for the Prevention of Disasters caused by Dangerous Substances, 1992; TNO Green Book, 1989). Este grado de intensidad de calor también significa aproximadamente un $1 \%$ de mortalidad para las personas expuestas durante 30 segundos antes de llegar a un refugio. Es por esto que normas como GRI00/0189, CFR-49-192 y NTC-5747 adoptan este nivel como admisible de radiación térmica en sus modelos de delimitación de las AAC. 


\section{Modelos de delimitación de AAC aplicables}

Modelos matemáticos de delimitación de AAC emitidos en normas nacionales e internacionales concluyen en una ecuación para calcular el radio de impacto potencial o el área de consecuencia, tal como se resume en la tabla 1, en la cual también se muestran los resultados de investigadores sobre el tema.

Tabla 1. Modelos matemáticos de cálculo del radio de impacto según investigadores y normas aplicables.

\begin{tabular}{|c|c|c|c|}
\hline \multicolumn{2}{|c|}{ Modelo matemático } & $\begin{array}{c}\text { Norma- } \\
\text { investigador }\end{array}$ & año \\
\hline \multicolumn{2}{|c|}{$r=0.685 \sqrt{p d^{2}}$} & GRI-00/0189 & 2000 \\
\hline \multicolumn{2}{|r|}{$A=a x^{b}$} & API-581 & 2002 \\
\hline \multicolumn{2}{|c|}{$r=0,69 * d * \sqrt{p}$} & NTC-5747 & 2009 \\
\hline \multicolumn{2}{|c|}{$r=0,69 * \sqrt{p * d^{2}}$} & CFR-49-192 & 2017 \\
\hline \multicolumn{2}{|c|}{$L=\sqrt{\frac{5 * x_{R} * \eta}{4 * \pi * Q_{G} * H_{G}}}$} & Huang y Li & 2012 \\
\hline \multicolumn{2}{|c|}{$r=10,285 \sqrt{Q}$} & $\begin{array}{l}\text { Hui y } \\
\text { Guoning }\end{array}$ & 2012 \\
\hline \multicolumn{2}{|c|}{$r=7,767 \sqrt{Q}$} & Ma y Li & 2013 \\
\hline
\end{tabular}

Fuente: elaboración propia.

Con las ecuaciones de los modelos matemáticos de las normas GRI, NTC y CFR se obtienen resultados muy similares; así como la función de la presión $p$ y el diámetro $d$ del tubo conductor.
La norma API-581 utiliza un modelo diferente que determina el área de consecuencia $(A)$; sin embargo, de ella se puede calcular el radio de un círculo de un área equivalente y de esa forma comparar los resultados. En este modelo a y $b$ son constantes que se extraen de tablas propias de la norma según el índice de escape $(x)$. Huang y Li presentan un modelo que considera la fracción de la radiación $\left(X_{R}\right)$, la eficiencia de la combustión $(\eta)$, el flujo de escape $\left(Q_{C}\right)$, el poder calorífico del gas $\left(H_{C}\right)$ y el número de fuentes $(n)$. En los demás modelos $Q$ es el índice de escape.

Es bien conocido que el modelo API es el más conservador. Con el fin de realizar una comparación entre los resultados de los diferentes modelos y que estos sean equiparables con los del modelo que se desarrolla en este trabajo, en la tabla 2 se muestran los resultados del radio de impacto, en metros, para un diámetro de fuga de 20 pulgadas y diferentes niveles de presión en el ducto.

\section{Desarrollo del nuevo modelo matemático de de- limitación de AAC}

El nuevo modelo matemático que se aplica mediante esta simulación relaciona el radio de impacto con la distancia a la estación de compresión. Para su desarrollo se consideró la localización geográfica y el trazado de un gasoducto tomado como caso de estudio. Para llegar a una ecuación del radio de impacto, primero se propone una ecuación que describa el cambio de la presión a lo largo de la conducción y con ella se plantea una ecuación

Tabla 2. Resultados comparativos del resultado del radio de impacto (m).

\begin{tabular}{|c|c|c|c|c|c|c|}
\hline \multicolumn{7}{|c|}{ Diámetro de fuga $=20$ pulgadas } \\
\hline Presión (psi) & API-581 & $\begin{array}{c}\text { NTC-5747 } \\
\text { CFR-49-192 }\end{array}$ & $\begin{array}{c}\text { GRI- } \\
00 / 0189\end{array}$ & Ma y Li & $\begin{array}{c}\text { Hui y } \\
\text { Guoning }\end{array}$ & Huang y Li \\
\hline 600 & 543 & 103 & 102 & 184 & $244^{\circ}$ & 135 \\
\hline 800 & 595 & 119 & 118 & 212 & 281 & 156 \\
\hline 1000 & 638 & 133 & 132 & 237 & 314 & 174 \\
\hline 1200 & 676 & 146 & 145 & 260 & 344 & 191 \\
\hline 1400 & 709 & 157 & 156 & 281 & 372 & 206 \\
\hline
\end{tabular}

Fuente: elaboración propia. 
para el índice de escape de gas en cualquier punto de la conducción. Finalmente, al considerar la intensidad de radiación térmica desde la llama en el punto de fuga, se determina la ecuación resultante del modelo matemático.

Trazado del gasoducto: para realizar la simulación del modelo de delimitación de AAC se propone un trazado que va desde Miraflores hasta Tunja. Este es una fracción del gasoducto Cusiana-Porvenir-La Belleza.

Caso de estudio: se considera el transporte de $80 \mathrm{~kg} / \mathrm{s}$ de gas natural por una conducción de diámetro constante de 20 pulgadas, constituida por tubo de acero sin costura con una rugosidad de 0,045 $\mathrm{mm}$ en un trayecto de 46,9 $\mathrm{km}$ de longitud, operando con una presión inicial de 1200 psi en la estación de compresión localizada en Miraflores y cuyo trazado se muestra en la figura 2.

En el desarrollo del nuevo modelo matemático que determina el radio de impacto potencial se considera el hecho que al disminuir la presión por efecto de la fricción del gas contra las paredes del tubo conductor, disminuye también la densidad lo que causa el aumento en la velocidad media de transporte.

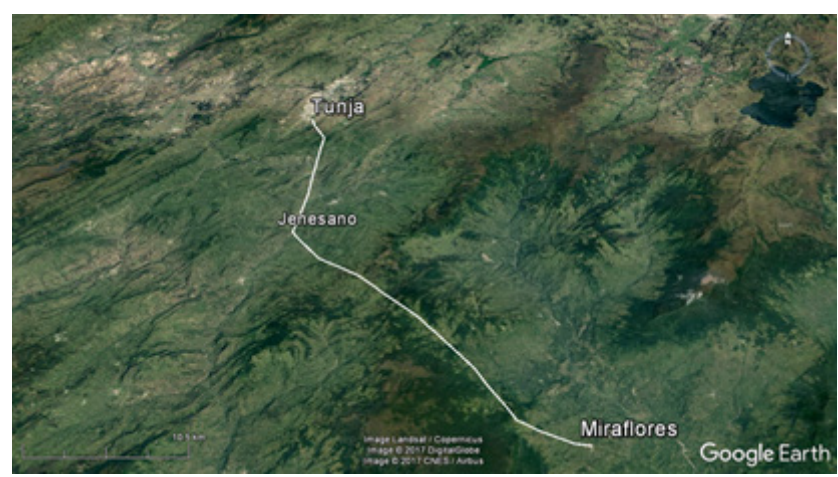

Figura 2. Trazado de gasoducto para caso de estudio.

Fuente: elaboración propia basada de Google Earth.

Para determinar los valores de la velocidad $(v)$, densidad $(\rho)$, temperatura $(T)$ y presión $(P)$ a lo largo de la conducción, se realiza un proceso iterativo dividiendo la longitud total del gasoducto en segmentos de análisis (para el caso de estudio cada $10 \mathrm{~km}$ ), de manera que las condiciones de salida de un segmento (subíndices 2) corresponden a las condiciones de entrada del segmento siguiente (subíndices 1). En este proceso iterativo se propone una velocidad de salida para el segmento. La densidad se determina con la ecuación de continuidad para conducción de fluidos por tuberías de sección constante (ecuación 1),

$$
\rho_{2}=\frac{\rho_{1} * v_{1}}{v_{2}}
$$

La temperatura se determina, entonces, con la ecuación de conservación de la energía para un proceso adiabático (ecuación 2), teniendo en cuenta que no hay interacciones de trabajo y el calor específico del gas (cp).

$$
T_{2}=T_{1}+\frac{\left(v_{1}^{2}-v_{2}^{2}\right)}{2 * c_{p}}
$$

La densidad promedio del segmento se calcula según la ecuación 3,

$$
\rho_{\text {prom }}=\frac{\left(\rho_{1}+\rho_{2}\right)}{2}
$$

La presión a la salida se determina mediante la ecuación de Darcy-Wellbach (4) donde f es el factor de fricción, I es la longitud del segmento y $D$ el diámetro del mismo,

$$
P_{2}=P_{1}-\frac{f * l * \rho_{\text {prom }} * v_{2}{ }^{2}}{D}
$$

El comportamiento del gas puede aproximarse al aplicar la ecuación de estado, donde $R$ es la constante del gas, afectada por el factor de compresibilidad $(z)$ y, entonces, se obtiene un nuevo valor de temperatura utilizando la ecuación 5,

$$
T_{2}{ }^{\prime}=\frac{P_{2}}{z * R * \rho_{2}}
$$

Entonces, cuando la diferencia entre la temperatura calculada según las ecuaciones 2 y 5 sea 
mínima, se termina el proceso iterativo con resultados para velocidad, densidad, temperatura y presión de salida del segmento. En la tabla 3 se muestran los resultados de la iteración y la caída de presión, en psi, según la distancia $L$ (en kilómetros) desde la estación de compresión para el caso de estudio, en donde se realizó un proceso iterativo hasta con tres decimales para la velocidad en una hoja de cálculo.

Tabla 3. Resultados de la iteración para los parámetros de operación en la conducción.

\begin{tabular}{ccccc}
\hline \multicolumn{5}{c}{ Propiedades del gas en la conducción } \\
$\mathbf{L}$ & $\begin{array}{c}\text { Densidad } \\
\mathbf{( k g / m 3 )}\end{array}$ & $\begin{array}{c}\text { Temperatura } \\
\mathbf{( K )}\end{array}$ & $\begin{array}{c}\text { Velocidad } \\
\mathbf{( m / s )}\end{array}$ & $\begin{array}{c}\text { Presión } \\
\mathbf{( p s i )}\end{array}$ \\
0 & 62,132 & 288 & 7,140 & 1200,00 \\
10 & 59,099 & 287,999 & 7,506 & 1141,48 \\
20 & 55,883 & 288,014 & 7,938 & 1079,41 \\
30 & 52,447 & 288,026 & 8,458 & 1013,01 \\
40 & 48.731 & 288,016 & 9,103 & 941,18 \\
50 & 44.641 & 287,998 & 9,937 & 862,19 \\
\hline
\end{tabular}

Fuente: elaboración propia.

Con el fin de efectuar el proceso de simulación usando Google Earth y Excel, se expresa mediante una ecuación exponencial la presión en función de la distancia desde la estación de compresión $\left(P_{L}\right)$, según la ecuación 6 . Una ecuación exponencial, como la propuesta, se puede hacer creciente o decreciente, es fácil de ajustar a una curva dada y de manipular en una hoja de cálculo.

$$
P_{L}=a * e^{b * L^{n}}
$$

Ajustando los parámetros $a, b$ y $n$ para que dado cualquier $L$ (en kilómetros) en el rango definido en la tabla 3 , se determine $P_{L}$ (en psi), la ecuación anterior queda según (7):

$$
P_{L}=1200 * e^{-0,001974 * L^{1,309}}
$$

En la figura 3 se muestra el descenso de la presión según la distancia L (en km) desde el punto de compresión, según los resultados de la tabla 3 (línea azul) comparados con los mismos obtenidos con la ecuación propuesta (línea naranja).

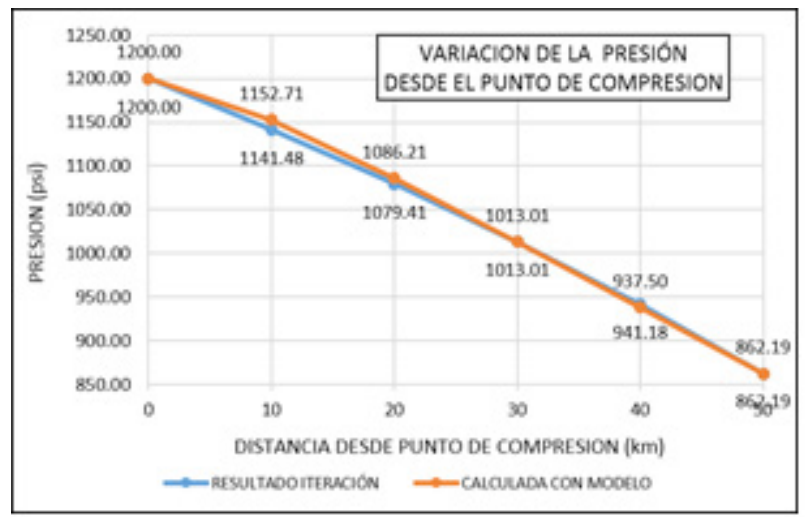

Figura 3. Variación de la presión desde la estación de compresión para el caso de estudio.

Fuente: elaboración propia.

Ahora, considerando un proceso de fuga isentrópico en el que las mayores consecuencias se tienen cuando el orificio de fuga es del mismo tamaño del diámetro del tubo conductor y que se tienen dos extremos en la rotura total, al utilizar los factores de conversión correspondientes la ecuación del índice de fuga $\left(Q_{\text {effect }}\right)$ en $\mathrm{kg} / \mathrm{s}$ a cualquier distancia $L$ en kilómetros para el caso de estudio se encuentra mediante la ecuación 8:

$$
Q_{\text {effect }}=1,243 * e^{-0,001974 * L^{1,309}}
$$

La intensidad de radiación (I) desde un chorro de fuego se expresa mediante la ecuación 9, la cual relaciona la eficiencia de combustión $(\eta)$, la emisibidad de la llama $\left(x_{g}\right)$, el índice de escape $\left(Q_{\mathrm{e}-}\right.$ ffect), el poder calorífico del gas $\left(H_{C}\right)$ y la distancia a partir del chorro $(r)$

$$
I=\frac{\eta * X_{g} * Q_{e f f e c t} * H_{c}}{4 * \pi * r^{2}}
$$

Se adopta un umbral de $15 \mathrm{~kW} / \mathrm{m}^{2}$ en el modelo en desarrollo como la intensidad de calor umbral con el fin de dimensionar un área de alta consecuencia por radiación térmica. De manera que, sustituyendo los valores y realizando las 
conversiones necesarias para determinar la distancia $r$ a la cual se alcanza este valor umbral desde el punto de fuga, se encuentra que la ecuación 10 es resultado del modelo matemático planteado en la que el radio de impacto potencial es función únicamente de la distancia a la estación de compresión, tal como sigue:

$$
r=151,9 * e^{-0,000987 * L^{1,809}}
$$

Donde:

$r$ es el radio de impacto potencial en metros.

$L$ es la distancia a la estación de compresión en kilómetros.

En la tabla 4 se presentan los resultados obtenidos para presión, índice de fuga y radio de impacto para el caso de estudio propuesto.

Tabla 4. Resumen resultados para $\mathrm{P}, \mathrm{Q}$ y $\mathrm{r}$ aplicando el modelo matemático.

\begin{tabular}{cccc}
\hline \multicolumn{4}{c}{ Resumen resultados modelo de delimitación AAC } \\
$\boldsymbol{L}(\mathbf{k m})$ & $\boldsymbol{P}_{\boldsymbol{L}}(\mathbf{p s i g})$ & $\boldsymbol{Q}_{\boldsymbol{L}}(\mathbf{k g} / \mathbf{s e g})$ & $\boldsymbol{r}(\boldsymbol{m})$ \\
0 & 1200,0 & 1,243 & 151,9 \\
10 & 1152,7 & 1,194 & 148,9 \\
20 & 1086,2 & 1,125 & 144,5 \\
30 & 1013,0 & 1,049 & 139,6 \\
40 & 937,5 & 0,971 & 134,3 \\
50 & 862,2 & 0,893 & 128,8 \\
\hline
\end{tabular}

Fuente: elaboración propia.

\section{Simulación del modelo de delimitación de AAC}

El punto de partida de la simulación es el trazado en Google Earth que se muestra en la figura 2. La localización del gasoducto del caso de estudio está conformada por una sucesión de puntos (13 en total) geo-referenciados que se extraen de Google Earth con un formato en grados decimales (coordenadas geográficas), contenidos en un archivo de extensión kml.

Las coordenadas del trazado se importan en un libro de cálculo en Excel y se almacena esta matriz de puntos para convertirlos en coordenadas cartesianas, en formato UTM, cuya ventaja es el tratamiento de distancias en unidades de longitud (metros o kilómetros) y rotaciones en grados o radianes.

En el libro de cálculo de Excel, mediante el diseño de macros, la segmentación original de 13 puntos del gasoducto se subdivide en tramos de menor magnitud que el radio mínimo de impacto. En cada punto de esta sub-segmentación se calcula el radio de impacto según la ecuación del modelo desarrollado y se constituye el área de impacto. De otra parte, se localizan geográficamente las áreas vulnerables de estudio y se calcula la distancia desde cada punto de la sub-segmentación hasta estas áreas, y si el resultado es menor al radio de impacto el área vulnerable se identifica como AAC.

\section{Descripción de las macros diseñadas en Excel para la simulación}

Para el caso de estudio se divide el gasoducto en tramos de máximo 100 metros, teniendo en cuenta que el radio de impacto mínimo (tabla 4) es de 128 metros. Particularmente, el trazado del gasoducto tiene una longitud de 46,9 kilómetros por lo que resultarán de la segmentación por lo menos 470 segmentos.

Entonces, para realizar la segmentación se leen las coordenadas de dos puntos consecutivos y se calcula la distancia $(d)$ entre estos puntos. $\mathrm{Si}$ la distancia es mayor a 100 metros se determina la parte entera de la relación entre la distancia dividida por 100 y un residuo y se le suma una unidad a la parte entera encontrada de manera que se establece en cuantos tramos de aproximadamente 100 metros de longitud ( $k=$ número de tramos) se divide el segmento tratado como lo muestran las ecuaciones 11 y 12 y se generan los puntos respectivos.

$$
d=\sqrt{\Delta X_{i}^{2}+\Delta Y_{i}^{2}}
$$




$$
k=\left(\text { entero }\left(\frac{d}{100}\right)+1\right.
$$

Si la distancia es menor a 100 metros no se hace segmentación y se continúa con el siguiente par de puntos como se muestra en el diagrama de bloques de la figura 4. En total, para el caso de estudio, se obtienen 474 segmentos, lo que representa que se generan 475 pares de puntos (474 +1 ) en coordenadas cartesianas que sustituyen a los 13 puntos iniciales, conservando la misma trayectoria.

Para generar el punto de la segmentación se inicia estableciendo la pendiente $\mathrm{m}$ y el ángulo $\theta$ formado con el eje $x$ por la línea recta que une el primer y segundo par de puntos del trazado, utilizando las ecuaciones 13 y 14.

$$
\begin{aligned}
& m=\frac{y_{2}-y_{1}}{x_{2}-x_{1}} \\
& \theta=\tan ^{-1} m
\end{aligned}
$$

La coordenada $X_{n}$ del siguiente punto de la sub-segmentación se obtiene al sumar al valor de la coordenada $X_{n-1}$ el producto de la longitud de la sub-segmentación por el coseno del ángulo formado, según la ecuación 15:

$$
X_{n}=X_{n-1}+l * \cos \theta
$$

La operación para encontrar la coordenada $X_{n}$ del segundo punto no experimenta consecuencias si la recta se encuentra en el primer y cuarto cuadrante, donde el coseno es positivo, pero da un resultado falso cuando se encuentra en el segundo y tercer cuadrante, donde el coseno es negativo. Es por eso necesario insertar un identificador $y$ un condicional según el sentido que tenga la recta para corregir el valor del ángulo $\theta$, tal como lo muestran las ecuaciones 16 y 17 :

$$
\operatorname{sentido}(x)=\left(x_{2}-x_{1}\right)
$$

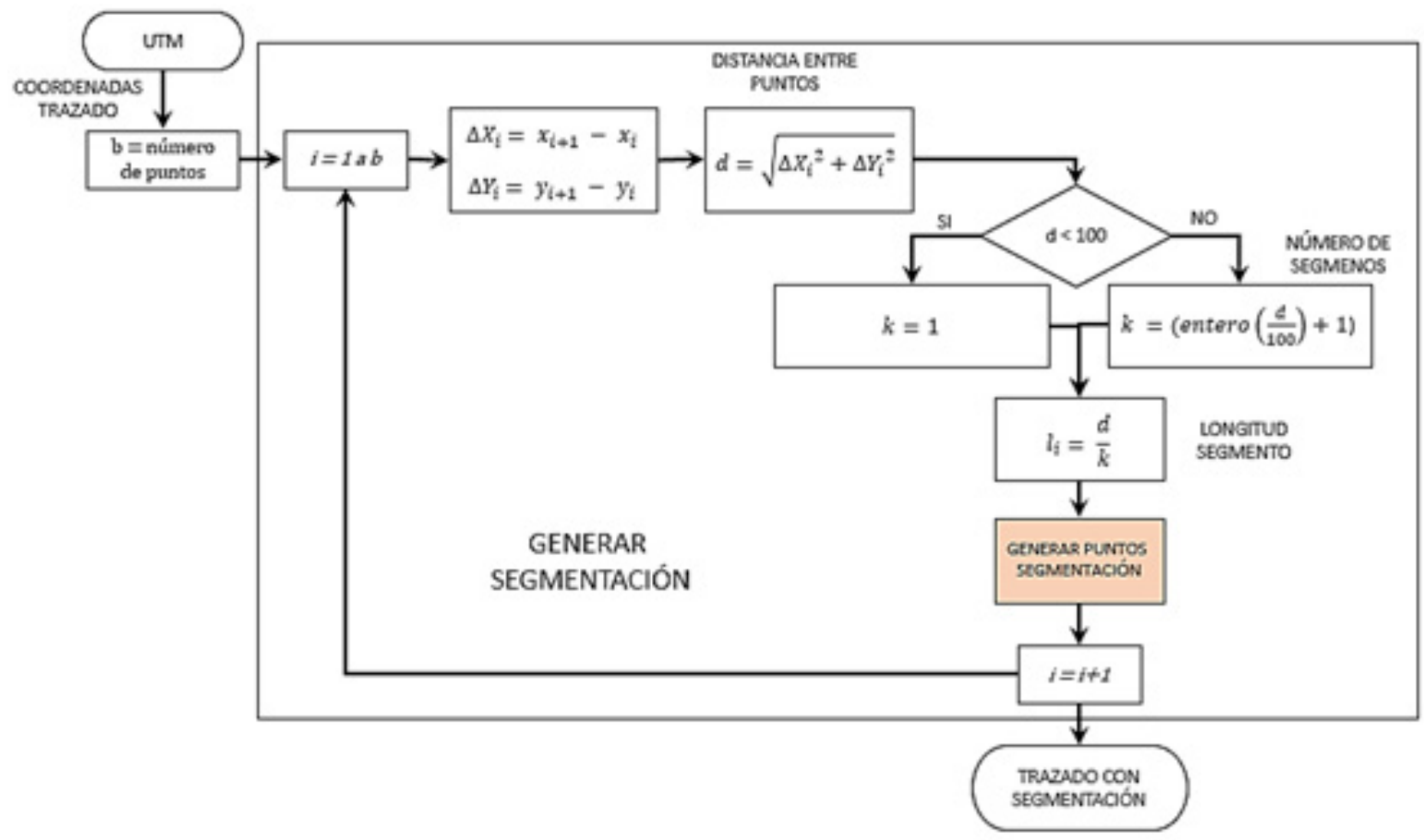

Figura 4. Diagrama de bloques para la segmentación del gasoducto.

Fuente: elaboración propia. 
si $(\operatorname{sentido}(x))<0$ entonces $(\theta=\pi+\theta)$ ysino $(\theta=\theta)$

Para obtener la coordenada $\mathrm{Yn}$ se realiza la operación que muestra la ecuación 18:

$$
Y_{n}=Y_{n-1}+m *\left(X_{n}-X_{n-1}\right)
$$

Esta rutina se repite $k$ veces en cada tramo original. El diagrama de bloques de este proceso se muestra en la figura 5 y de esta forma se obtienen las coordenadas $\left(X_{n}, Y_{n}\right)$ de cada punto de la sub-segmentación.

El área de impacto potencial es una franja alrededor del gasoducto de ancho igual al diámetro de impacto potencial (dos veces el radio) con centro en el trazado. Para cualquier punto del trazado del gasoducto se calcula el radio de impacto según la ecuación del modelo matemático desarroIlado en este trabajo (ecuación 10). La distancia $L$ se obtiene al acumular las longitudes en que se ha dividido el gasoducto hasta el punto en análisis. La franja se construye con dos líneas paralelas al trazado y a una distancia $r$ del trazado. Estas líneas a su vez están conformadas por puntos situados sobre la línea perpendicular a la línea del trazado; entonces, si las coordenadas del punto del trazado son $\left(X_{n}, Y_{n}\right)$, las coordenadas de los puntos de la línea arriba y a la derecha del trazado se determinan con las ecuaciones 19 y 20.

$$
\begin{gathered}
X_{n^{\prime}}=X_{n}-r * \cos \left(\frac{\pi}{2}-\theta\right) \\
Y_{n^{\prime}}=Y_{n}+r * \cos \theta
\end{gathered}
$$

Y para la línea abajo y a la izquierda del trazado, las coordenadas se determinan con las ecuaciones 21 y 22 .

$$
\begin{gathered}
X_{n^{\prime \prime}}=X_{n}+r * \cos \left(\frac{\pi}{2}-\theta\right) \\
Y_{n^{\prime \prime}}=Y_{n}-r * \cos \theta
\end{gathered}
$$

En la figura 6 se muestra el diagrama de bloques de este proceso para construir el área de impacto.

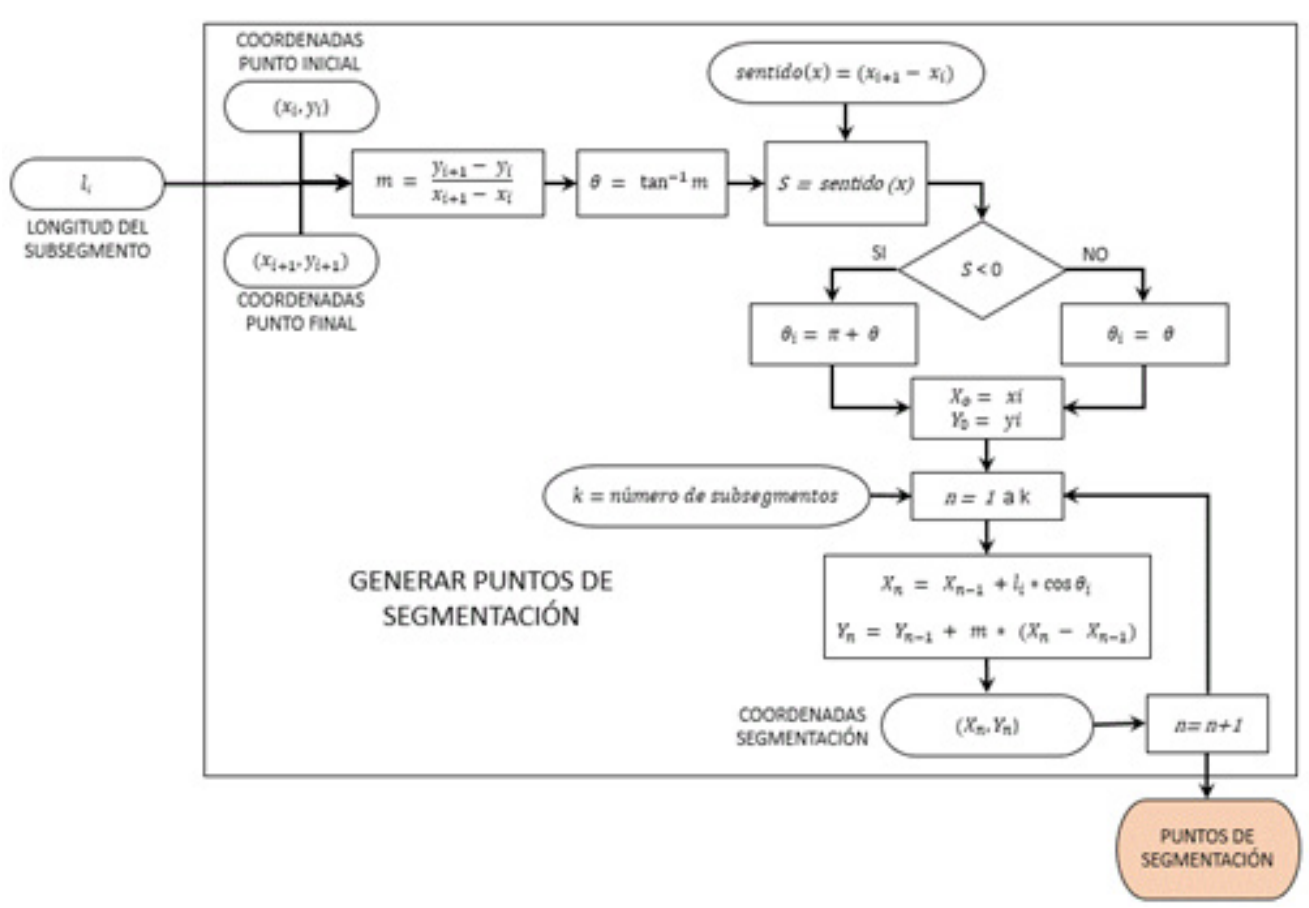

Figura 5. Diagrama de bloques para generar puntos de segmentación.

Fuente: elaboración propia. 


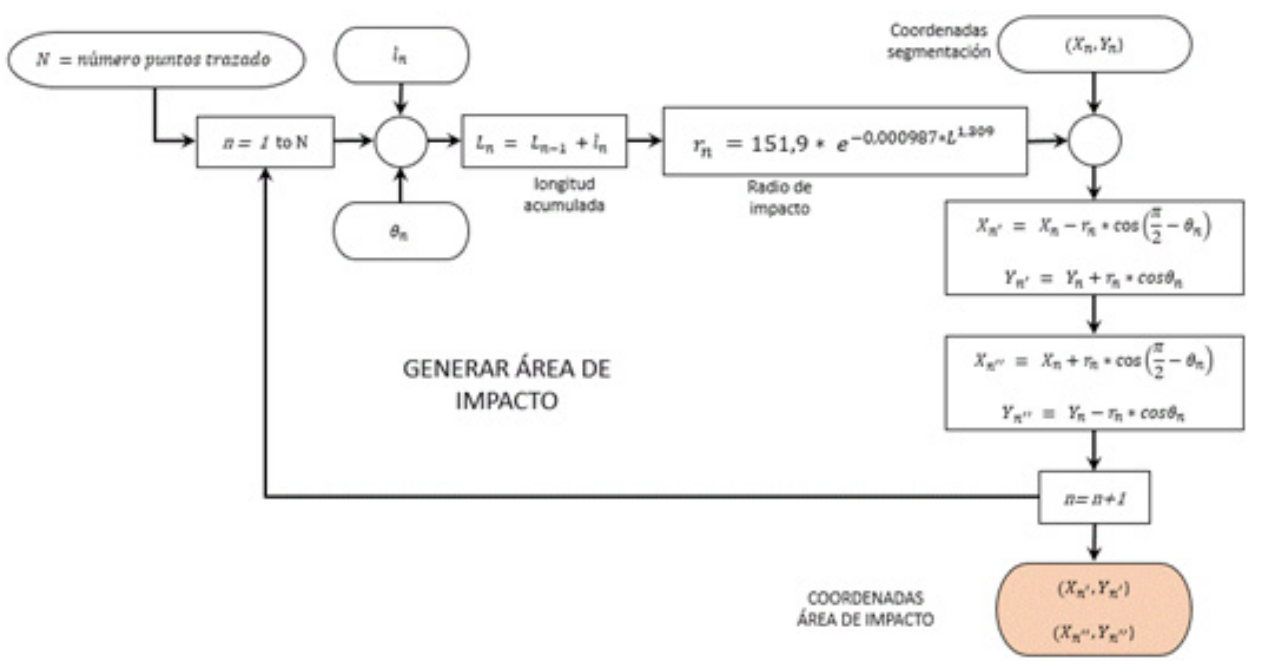

Figura 6. Diagrama de bloques para generar el área de impacto.

Fuente: elaboración propia.

Mediante una revisión del área circundante al gasoducto se indican las coordenadas de las áreas vulnerables, cargándolas en una nueva matriz de datos los cuales también se cambian del formato geográfico a UTM con el fin de realizar el cálculo de la distancia desde los puntos en que se ha segmentado el gasoducto y estas localizaciones.

Las AAC corresponden a las zonas donde se intersectan las áreas vulnerables con el área de impacto. Entonces, utilizando la geo-referenciación realizada para los k puntos vulnerables, en donde estarán ubicados según coordenadas $\left(X_{k}, Y_{k}\right)$ , se calcula la distancia a la que se encuentra este punto con cada punto del trazado utilizando la expresión 22.

$$
D=\sqrt{\left(\left(X_{n}-X_{k}\right)^{2}+\left(Y_{n}-Y_{k}\right)^{2}\right.}
$$

Se compara esta distancia D con el radio de impacto $r$ de cada punto del trazado en particular y, si se cumple la condición de la ecuación 23, entonces el área sensible se identifica como AAC y el punto del trazado del gasoducto se identifica como tramo del gasoducto que afecta AAC.

$$
D<r
$$

Si esta condición no se cumple para ningún punto del trazado, el área sensible está por fuera del área de impacto y no afecta las AAC, como se muestra en el diagrama de bloques que se sigue en la macro en Excel de la figura 7.

\section{Resultados}

Con el modelo de simulación desarrollado, al visualizar el área de impacto en el Google Earth se aprecian por simple inspección las AAC, según se localicen las áreas vulnerables dentro o fuera de ella. Sin embargo, una vez que se analizan las áreas vulnerables, se colocan dos identificadores en el libro de cálculo: el primero marca las áreas vulnerables que son AAC y el segundo marca el punto del gasoducto que afecta AAC, siendo ambos apreciables en el resultado final.

En el simulador desarrollado, las coordenadas cartesianas de los puntos del trazado, las áreas vulnerables analizadas, el área de impacto y los tramos del gasoducto que afectan AAC se cambian a coordenadas geográficas para ser visualizadas en Google Earth, como se muestra en la figura 8. En esta se extrajo un pequeño cuadrante del trazado mostrando la identificación del gasoducto (línea 


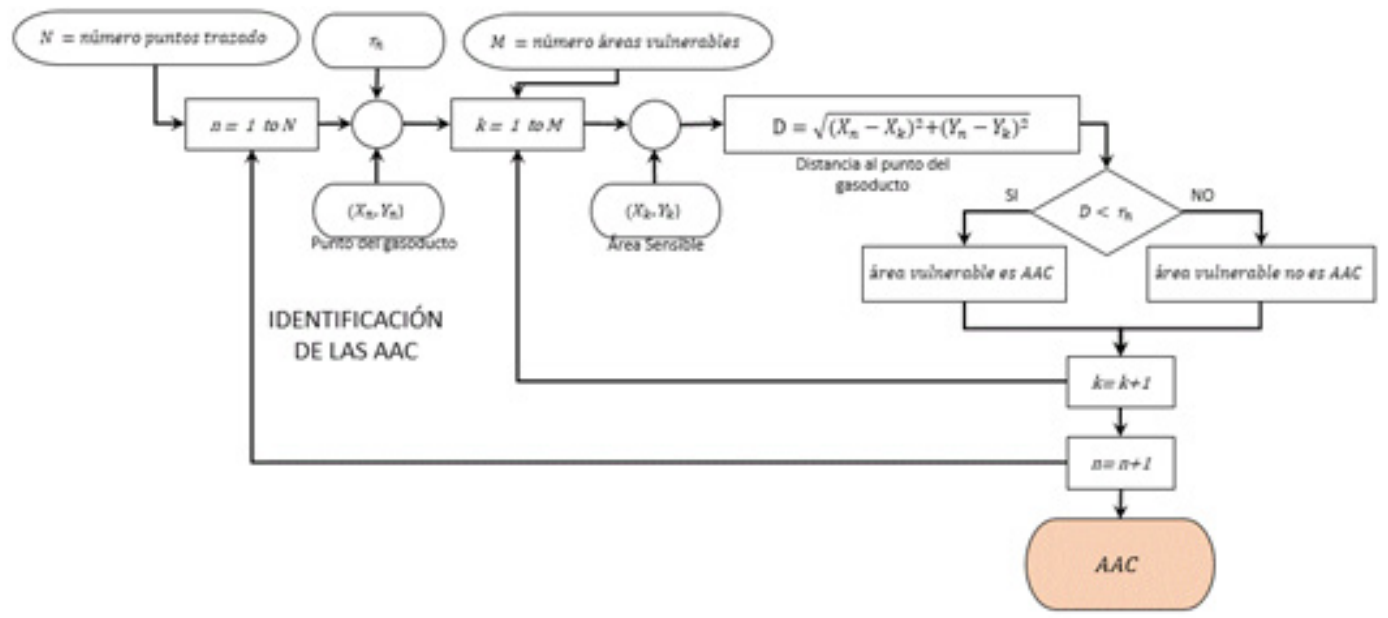

Figura 7. Diagrama de bloques para la delimitación de las AAC.

Fuente: elaboración propia.

blanca), el área de impacto (zona roja traslucida), área sensible (casas 353, 360 y 368), los tramos de gasoducto que afectan AAC (puntos rojos del trazado) y los segmentos que no afectan AAC (puntos verdes).

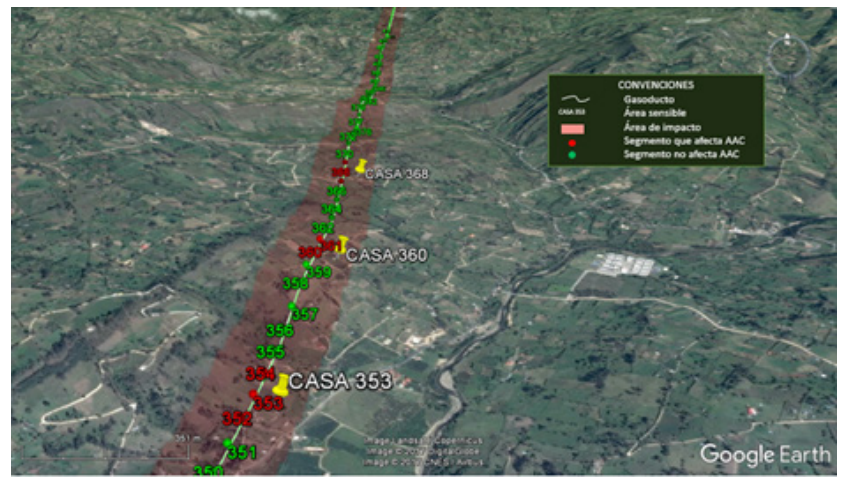

Figura 8. Cuadrante del gasoducto donde muestra el resultado de AAC.

Fuente: elaboración propia badasa en Google Earth.

La numeración que se aprecia en la figura 8 (351 a 372) corresponde a los números asignados a cada punto en que se sub-segmentó el trazado en tramos distanciados menos de 100 metros. Los puntos rojos e identificados con los números 352, 353 y 354 son tramos del gasoducto que afectan AAC. Los demás puntos se muestran en color verde, lo que indica que los tramos del gasoducto entre ellos no afectan AAC. La franja del área de impacto observada tiene la dimensión del radio de impacto calculado con el modelo matemático desarrollado.

\section{Interfaz para la simulación de las AAC}

En la figura 9 se muestra la interfaz desarrollada en Excel con la que se realiza la delimitación de las AAC y se obtienen los resultados mostrados en la figura 8.

Una vez se ha realizado la simulación en Excel, se genera un archivo kml para ser leído en Google Earth y visualizar los resultados de la simulación. El archivo generado es guardado en la carpeta que se señala en las cajas de selección. Al final se tendrán dos archivos: un libro de cálculo en Excel con las ejecuciones de las macros descritas y realizadas y un archivo $\mathrm{kml}$ para visualizar los resultados en Google Earth.

\section{Conclusiones}

El modelo matemático desarrollado en este trabajo delimita las AAC mediante una ecuación exponencial de tres constantes y una única variable, 
que es la distancia a la estación de compresión. El modelo matemático se evaluó frente a otros modelos aplicables correspondientes a normas nacionales e internacionales y a las conclusiones de otros trabajos desarrollados por investigadores a nivel mundial. Los resultados se validan al estar comprendidos entre los modelos más y menos conservadores, pero cercanos a estos últimos, lo que representa una ventaja para el operador del gasoducto al considerar también la disminución de la presión con la distancia a la estación de compresión.

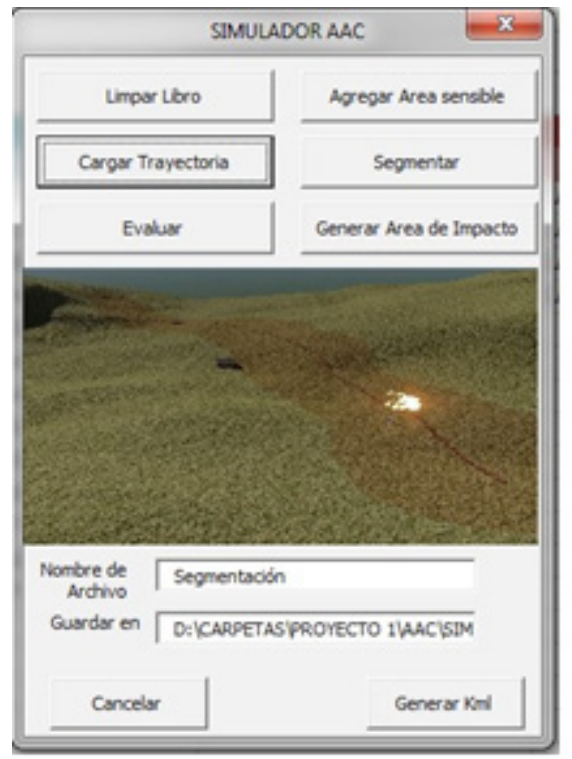

Figura 9. Interfaz para simulación del modelo de AAC.

Fuente: elaboración propia.

El modelo desarrollado se utiliza para determinar el radio de impacto potencial y tiene en cuenta la variación de la presión, el índice de escape de gas y un umbral de radiación admisible. También considera las propiedades del gas transportado, las características geométricas del tubo conductor, los parámetros de operación del gasoducto, el trazado del mismo y la localización de áreas vulnerables alrededor del trazado. Es de especial relevancia en un estudio de AAC la disminución de la presión al interior del tubo conductor debida a los efectos de la fricción y que son consideradas en este modelo.
La simulación del modelo matemático se realizó mediante el diseño de macros para la ejecución de procesos en lazo cerrado utilizando una hoja de cálculo de Excel, de manera que al exportar los resultados se obtiene la visualización de las AAC y de los tramos de gasoducto que afectan AAC mediante un software de amplia utilización que puede ser manejado por personal de poca experiencia como Google Earth, representando una fortaleza adicional para su utilización.

El modelo matemático y la simulación se aplicaron a un caso de estudio y puede ser aplicado en cualquier otro, para lo cual deben ajustarse los valores de los parámetros $\mathrm{a}$, b y $\mathrm{n}$ de la ecuación de la presión a lo largo del gasoducto y con ello obtener los nuevos parámetros de la ecuación exponencial del radio de impacto. También se requiere conocer el trazado para trasladar las coordenadas geográficas al libro en Excel y realizar el tratamiento correspondiente a la sub-segmentación y generación del área de impacto.

\section{Agradecimientos}

Este trabajo contó con la participación efectiva de diversas entidades de la Universidad Pedagógica y Tecnológica de Colombia, tales como la Vicerrectoría de Investigación y Extensión, el grupo de Geomática de la Escuela de Ingeniería Civil, los docentes de la Escuela de Ingeniería Electromecánica y los integrantes de los grupos de investigación Giem y Gente.

\section{Referencias}

API-581. (2002). Risk-Based Inspection Base Resource Document. American Petroleum Institute, Washington, DC.

Code of Federal Regulations, US. (2017). Title 49Part 192-Transportation of Natural and Other Gas by Pipeline: Minimum Federal Safety Standards. En: Code Of Federal Regulations.

Committee for the Prevention of Disasters caused by Dangerous Substances. (1992). Green Book 
Methods for the Determination of Possible Damage Methods for the Deternination of Possible Damage. [CPR 16E].

Daza-leguizamón, O., Vera-lópez, E. y Riaño-Cano, G. (2016). Efecto de la resolución espacial de modelos digitales de elevación en la simulación de derrames de hidrocarburos. Revista Científica, 3(26), 149-72. https://doi. org/10.14483/23448350.11620

GRI-00/0189. (2000). A Model for Sizing High Consequence Areas Associated with Natural Gas Pipelines. Alberta, Canadá: Gas Research Institute.

Huang, Z. y Li, J. (2012). Assessment of Fire Risk of Gas Pipeline Leakage in Cities and Towns. Procedia Engineering, 45, 77-82. https://doi. org/10.1016/j.proeng.2012.08.124

Hui, S. y Guoning, D. (2012). Risk Quantitative Calculation and ALOHA Simulation on the Leakage Accident of Natural Gas Power Plant. Procedia Engineering, 45(4), 352-59.

Jo, Y. y Ahn, B. (2002). Analysis of Hazard Areas Associated with High-Pressure Natural-Gas Pipelines. Journal of Loss Prevention in the
Process Industries, 15, 179-88. https://doi. org/10.1016/S0950-4230(02)00007-4

Jianhuaa, L. y Zhenghua, H. (2012). Fire and Explosion Risk Analysis and Evaluation for LNG Ships. Procedia Engineering, 45, 70-76. https:// doi.org/10.1016/j.proeng.2012.08.123

Ma, L. Yongshu, L., Lei L., Manchun L. y Liang C. (2013). A Novel Method of Quantitative Risk Assessment Based on Grid Difference of Pipeline Sections. Safety Science, 59, 219-226. DOI: http://dx.doi.org/10.1016/j.ssci.2013.04.012

NTC-5747. 2009. "Gestión de Integridad de Gasoductos." Editorial ICONTEC. pp. 107

Park, K.S., Lee, J.H. y Jo, Y.D. (2004). An Approach to Risk Management of City Gas Pipeline. Process Safety and Environmental Protection, 82, 446-452. https://doi.org/10.1205/ psep.82.6.446.53210

Tong, S.J., Siu, M.L., Pei H.Z. y Bao, Z.C. (2013). Jet Fire Consequence Evaluation on the $\mathrm{Na}$ tural Gas Transported by Pipelines. Procedia Engineering, 52, 349-54. http://dx.doi.org/10.1016/j.proeng.2013.02.152. 